\title{
METHOTREXATE IN PATIENTS WITH TAKAYASU'S ARTERITIS: A RETROSPECTIVE COHORT
}

Eduardo Florim Terra ${ }^{1, \star}$, Pedro Serra de Candol', Taíssa Cerqueira dos Santos ${ }^{1}$, Juliana Murad Faria ${ }^{1}$, Carlos Eduardo Duek Souza ${ }^{1}$, Melissa Adriana Torres Gonzalez ${ }^{1}$, Camila Souto Oliveira Elias ${ }^{1}$, Leticia Rocha Pereira ${ }^{1}$, Manuella Lima Gomes Ochtrop ${ }^{1}$

1.Universidade do Estado do Rio de Janeiro, Rio de Janeiro (RJ), Brazil.

*Corresponding author: eduardofterra@hotmail.com

\section{BACKGROUND}

Takayasu's arteritis (TA) is a large-vessel vasculitis involving primarily the aorta and its major vessels. Corticosteroids remains the first-line therapy in TA. Methotrexate (MTX) is a disease modifying anti-rheumatic drug (DMARD) widely used to minimize corticosteroid exposure and achieve disease remission. Data regarding MTX use in this context is limited. The aim of this study was to describe clinical characteristics of a retrospective cohort of TA patients using MTX.

\section{METHODS}

Patients fulfilling the 1990 American College of Rheumatology (ACR) classification criteria for TA and with documented use of MTX were included. Demographic, clinical, laboratorial and imaging data were also collected from medical records. Data on MTX use, dose and reason for discontinuation were collected at baseline and at 6.12 and 18 months. Clinical assessment of disease activity based on the National Institutes of Health $(\mathrm{NIH})$ criteria, erythrocyte sedimentation rate (ESR) and prednisone dose were also collected for the follow-up period (FUP). A descriptive analysis was performed, presenting measures of central tendency and dispersion according to each variable distribution.

\section{RESULTS}

Among 54 patients under regular FUP in our unit, 24 (44\%) patients fulfilled the inclusion criteria; of those, 23 (95.8\%) were female, with median disease duration of 17 years (range: 4-43 years) and 14 (54\%) presented angiographic classification type $V$. At the end of the 18-month FU period, 18 (75\%) patients were still on MTX, with the remaining 6 (25\%) having discontinued the DMARD due to treatment failure or intolerance. Within those 6 patients, 1 suspended MTX at the 6 -month evaluation, 4 at the 12 -month FUP and 1 at 18-month FUP. At the 18-month FUP, 6 (33\%) patients remained in clinical remission, although data on disease activity from 5 patients were unavailable at this timepoint. The median daily dose of prednisone and ESR at baseline was $40 \mathrm{mg}$ (range: 5-60, $\mathrm{n}=22$ ) and $31 \mathrm{~mm} / \mathrm{h}$ (range: $4-120, \mathrm{n}=21$ ), respectively. At the 18-month FUP, median prednisone daily dose was $5 \mathrm{mg}$ (range: $2.5-20, n=13$ ) and ESR was $28 \mathrm{~mm} / \mathrm{h}$ (range: $9-80, n=13$ ), respectively.

\section{CONCLUSION}

Despite showing a considerable glucocorticoid sparing effect at 18 months, $25 \%$ of patients had suspended MTX therapy at this time point and only $33 \%$ of those using the DMARD remained in remission. These data are in accordance with a recently published metanalysis that found a proportion of patients attaining at least partial clinical response from $50 \%$ during MTX therapy.

\section{KEYWORDS}

Takayasu, Methotrexate, Prednisone, Remission. 